\title{
ALIQUOTING STRUCTURE FOR CENTRIFUGAL MICROFLUIDICS BASED ON A NEW PNEUMATIC VALVE
}

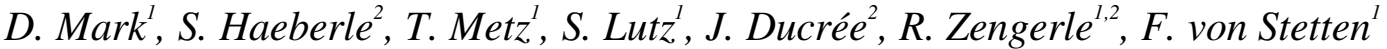 \\ ${ }^{1}$ Laboratory for MEMS Applications, Department of Microsystems Engineering (IMTEK), \\ University of Freiburg, Georges-Koehler-Allee 106, D-79110 Freiburg, Germany \\ ${ }^{2}$ HSG-IMIT, Wilhelm-Schickard-Straße 10, D-78052 Villingen-Schwenningen, Germany
}

\begin{abstract}
We present a new microvalve that can be monolithically integrated in centrifugally driven lab-on-achip systems. In contrast to existing operation principles that use hydrophobic patches [1], geometrically defined capillary stops [2] or siphons [3], here we present a pneumatic principle. It needs neither additional local coatings [1] nor expensive micro sized geometries [2]. The valve is controlled by the spinning frequency and can be switched to be open when the centrifugal pressure overcomes the pneumatic pressure inside an unvented reaction cavity. We designed and characterized valves ranging in centrifugal burst pressure from $6700 \mathrm{~Pa}$ to $2100 \mathrm{~Pa}$. Based on this valving principle we present a new structure for aliquoting of liquids. We experimentally demonstrated this by splitting $105 \mu \mathrm{L}$ volumes into 16 aliquots with a volume $\mathrm{CV}$ of $3 \%$.
\end{abstract}

\section{INTRODUCTION}

Centrifugal microfluidics is an increasingly popular tool for the development of biological and medical diagnostic platforms. The great advantage over traditional analysis and diagnostic tools lies in its potential for automation and parallelization at minimal costs as well as in its potential as point-of-care platform [4]. Recent developments include platforms for automated nucleic acid analysis [5] and immunoassays. However, most of the systems suffer from a certain complexity of the (disposable) test cartridge often associated with the valving of fluids. This includes local hydrophobic patches [6] which complicate the mass production and potentially limit the shelf-live. Other platforms make use of expensive sacrificial valves [7]. However, to compete with existing convential diagnosis platforms on the market, the price for each test has to be minimized. Here we present a new valving concept that simply relies on air pressure inside an unvented chamber and is straightforward to mass produce with established technologies such as injection molding.

As an application of the novel valve we developed a new aliquoting structure (Fig. 1). This permits splitting of a sample into several reaction chambers with pre-stored lyophilized reagents. The volumes of the aliquots are independent of the volume of pre-stored substances. The structure provides excellent fluidic separation between the aliquots to avoid any cross-contamination.

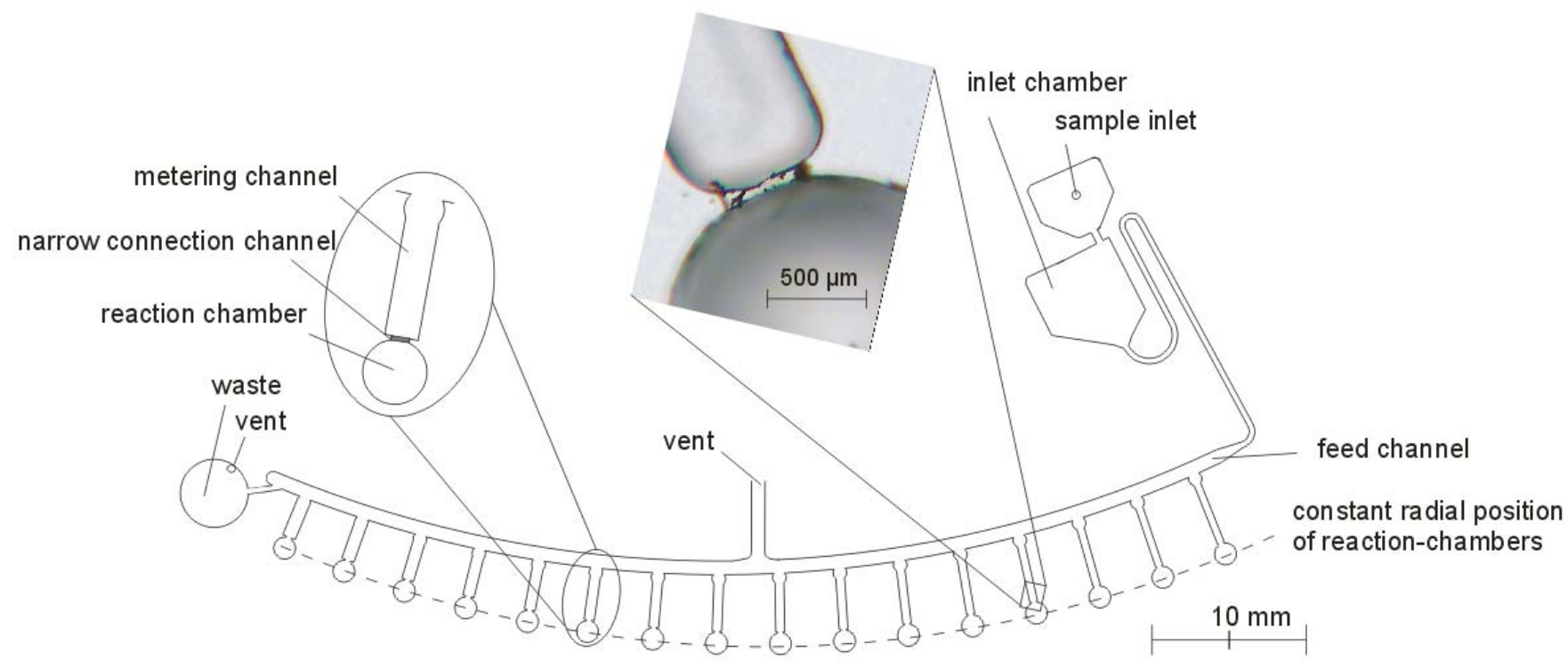

Figure 1: Sketch of the aliquoting structure. The feed channel is slightly inclined with respect to a circular segment (dashed line) to promote centrifugal filling and overflow. The metering channels all accommodate the same volume. They are connected to the reaction chambers via a narrow connection channel to guide the fluid during priming. The equiradial positions of the detection chambers facilitate optical detection. The rectangular image in the top center shows a microscopic image of the micro-milled narrow connection channel. 

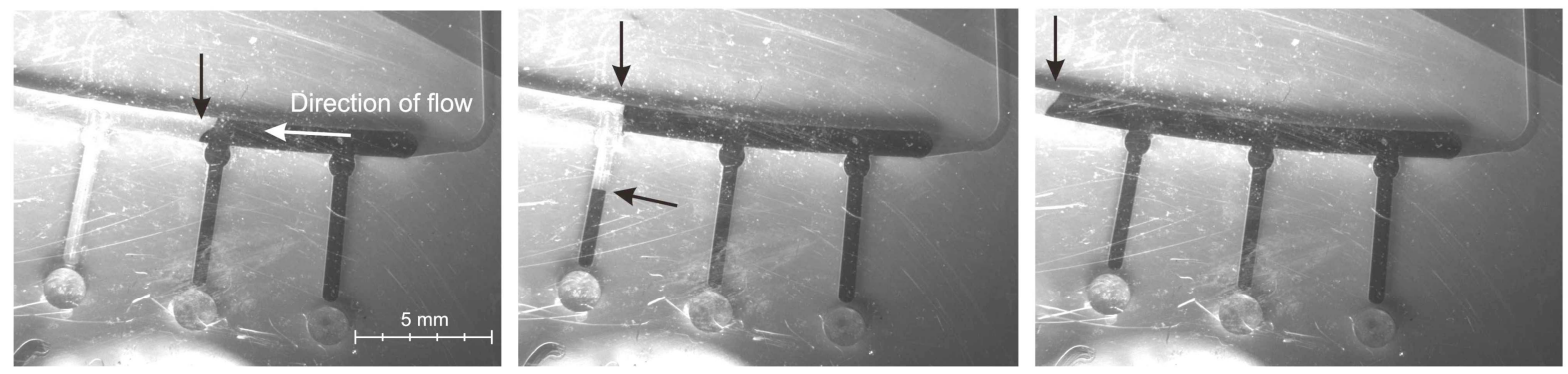

Figure 2: Image series: Filling of the metering channel with ink stained water (dark). The arrows indicate the menisci in the feed channel and metering channels. The circular reaction chambers remain dry at this frequency.

\section{WORKING PRINCIPLE Design}

The pneumatic valve consists of an upstream metering channel and a downstream unvented reaction chamber separated by a narrow connection channel (Fig. 1). Fluid entering the metering channel seals the reaction chamber by forming a liquid plug, since the narrow connection channel does not allow spontaneous venting (Fig. 2). When the rotational speed is increased, the meniscus from the liquid plug increasingly protrudes into the reaction chamber before it abruptly disintegrates to clear a bypath for part of the entrapped air, thus releasing liquid into the reaction chambers (Fig. 3)
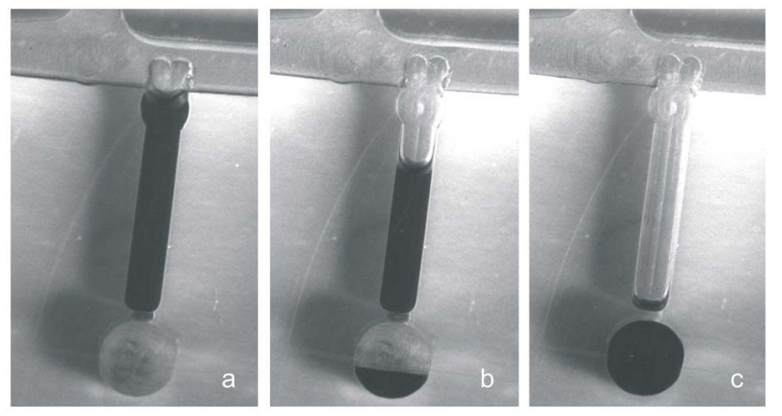

Figure 3: a) Ink-colored fluid stops at the connection channel, b) after increasing the rotational speed, the metering channel starts filling the reaction chamber and c) finally fills the reaction chamber completely.

The test structures which are at the same time aliquoting structures are made of a slightly sloping feed channel from which 16 side channels with pneumatic valves branch off (Fig. 1). To guarantee uniform distribution of metered volumes and enable optical readout of reactions at a constant radial position, the radial compression of the metering sections is compensated by a lateral widening.

In a first phase at low rotational frequencies $(\sim 10 \mathrm{~Hz})$, the metering channel fills with fluid that displaces the air in this channel towards the feed channel. The liquid stops at the pneumatic valve. Continuing rotation, excess liquid overflows into the waste, thus defining metered volumes. In the second phase the rotational frequency is elevated to about $25 \mathrm{~Hz}$. This releases the metered volumes into the reaction chambers.

The two-stage principle of metering volumes first and transporting them into a reaction chamber in a second step guarantees that no cross-contamination between the reaction chambers occurs and that pre-stored reagents are dissolved simultaneously in all reaction chambers. Additionally, the metered volumes are independent of pre-stored reagents.

\section{Theory of the pneumatic valve}

Effects related to the instability of an interface between two media in a gravitation-like field are commonly referred to as Rayleigh-Taylor instabilities. In general, if the media with the higher density is situated above the media with the lower density, this interface is unstable. Small perturbations of the interface lead to growing "bubbles", finally resulting in the heavier media sinking below the lighter media. However, perturbations with a wavelength smaller than a critical wavelength $\lambda_{\text {crit }}$ are stabilized by the surface tension [8]:

$$
\lambda_{\text {crit }}=2 \pi\left[\sigma / G\left(\rho_{\mathrm{H}}-\rho_{\mathrm{L}}\right)\right]^{1 / 2},
$$

where $\sigma$ is the surface tension, $\rho_{\mathrm{H}}$ and $\rho_{\mathrm{L}}$ are the densities of the heavier and lighter medium and $G=\left(2 \pi v_{\text {burst }}\right)^{2} r$ is the effective acceleration of the system with $v_{\text {burst }}$ the burst frequency of the valve and $r$ the mean radial position of the liquid plug. This formula is an approximation for a plane interface and incompressible media. However, a next order approximation shows that the effects of compressibility are minor [9].

The critical wavelength depends on the spinning frequency. As a rough estimate one would expect bursting of the valve for a critical wavelength that is about two times the diagonal diameter of the connection channels cross section. This should be the largest wavelength of surface waves that can be stimulated at the liquid gas interface. Nevertheless, we observed bursting of the valve for critical wavelengths of about four times the diameter of the connection channel (Fig. 4). This may be a result of the curved liquid gas interface that enables longer wavelengths to be stimulated. The actual shape of the interface and therefore the largest wavelength are not easily described analytically. However, the number of four is assumed as an upper limit for the ratio between critical wavelength and connection channel diameter for this geometry. It should permit the prediction of burst frequencies from channel geometries. 


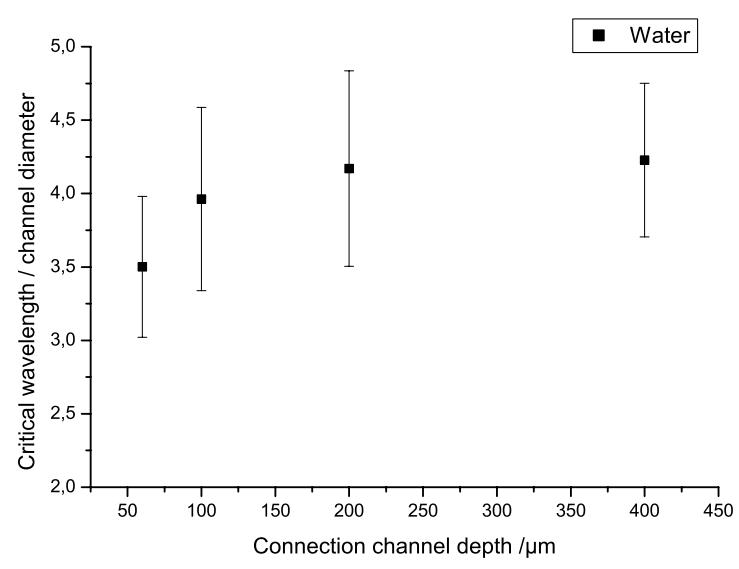

Figure 4: Ratio of the critical wavelength to the connection channel diameter for different connection channel depths. The bars show the standard deviation. Each value is calculated from 16 data points.

\section{Simulation of the filling process}

Since the actual filling process of the reaction chamber is difficult to observe, we used a numerical computational fluid dynamics (CFD) simulation to view the process (Fig. 5). It shows that after the fluid meniscus ruptures from the liquid plug, the reaction chamber is partially vented by an air bubble. While the bubble escapes from the chamber, fluid enters it through the corners of the connection channel.
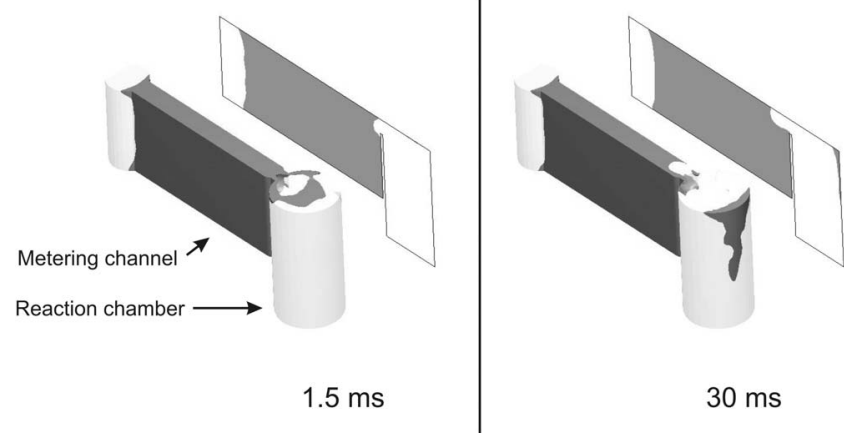

Figure 5: CFD simulation of the valve. At $1.5 \mathrm{~ms}$, the meniscus ruptures from the liquid plug and fills the chamber. At $30 \mathrm{~ms}$, the chamber is partially vented by formation of an air bubble. The 2D images show crosssections through the center of the channel.

\section{FABRICATION}

The aliquoting structure was micro-milled in a cycloolefin-polymer (COP) disc. To render the channels hydrophilic, they were coated with poly-ethylene-glycol (PEG, molecular weight 15-20 kD). The disk was sealed with a two-layered foil as described in [10].

\section{EXPERIMENTS AND RESULTS}

The measurements were carried out with ink-stained water at metering channel lengths between $3.08 \mathrm{~mm}$ and
$5.36 \mathrm{~mm}$ and connection channel depths varying between 60 and $400 \mu \mathrm{m}$. Centrifugal burst pressures $p_{\text {burst }}$ were calculated from burst frequencies $v_{\text {burst }}$ according to

$$
p_{\text {burst }}=\left(2 \pi v_{\text {burst }}\right)^{2} r l \rho,
$$

( $l=$ length of liquid plug, $r=$ mean radial position, $\rho=$ density of water). The valve showed burst pressures between $6700 \mathrm{~Pa}$ and $2100 \mathrm{~Pa}$ at CVs between $28 \%$ and $37 \%$ (Fig. 6) associated with tolerances of the milling process.

To explicitly proof the pneumatic principle, venting holes were created in the reaction chambers that prohibit building up a pneumatic pressure. This reduced the burst pressures by a factor 4 or more compared to original structures (Fig. 6), clearly demonstrating the pneumatic valve principle. In addition we proved the minor influence of capillary forces by adding the detergent Tween $20(0.1 \% \mathrm{v} / \mathrm{v})$ to reduce surface tension. The burst pressures were reduced compared to water, as formula 1 implies, but the fluid could still be switched reliably (Fig. 6).

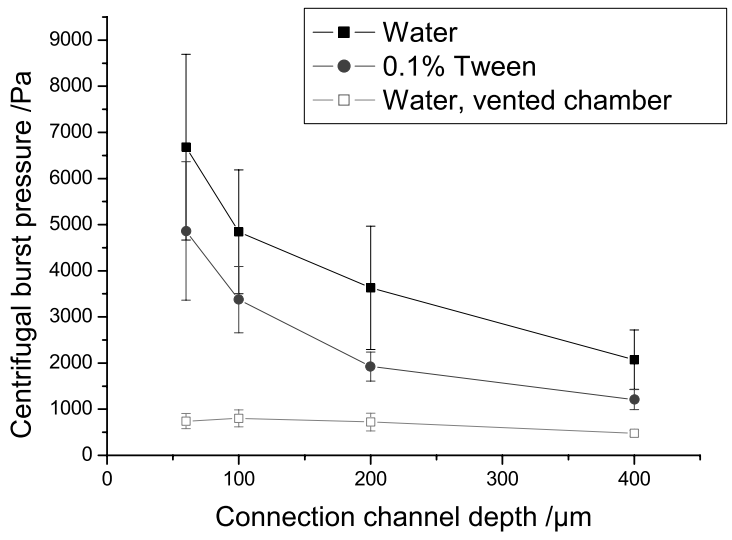

Figure 6: Dependency of the centrifugal burst pressure on the depth of the narrow connection channel. The bars show the standard deviation. Burst frequencies have been measured for water and water with $0.1 \% \mathrm{v} / \mathrm{v}$ Tween 20 . The low burst pressures for vented reaction chambers clearly show that capillary stopping effects are negligible compared to the effect of the pneumatic pressure. All values were calculated from at least 8 data points.

To evaluate the precision of the fluidic metering structure, we used a design with larger, elongated reaction chambers that allow a more precise measurement of eventual filling levels. 25 values obtained from aliquoting of $105 \mu 1$ samples were measured. The eventual liquid levels were measured as captured by a camera (Fig. 7). The first and last reaction chamber showed significantly higher and lower filling levels, respectively and were not included in the calculation. The reason for the lower filling level in the last chamber is probably associated with creeping of fluid into the waste chamber. The first channel shows higher filling levels since it bursts first and collects residual fluid from the feed channel. These effects can be avoided by placing a "sacrificial" chamber that has a lower burst frequency and is placed next to 
the last chamber. This chamber could also replace the waste chamber. The mean filling level of the reaction chambers without the first and last chamber was measured to be $2.8 \mathrm{~mm}$ with a CV of $3 \%$ (see Fig. 8). The fluid volume is around $6 \mu \mathrm{l}$ in each chamber but is difficult to calculate exactly because of the menisci at the chamber walls However, the $\mathrm{CV}$ of $3 \%$ demonstrates the good suitability of the valve and the design as a precise aliquoting structure.

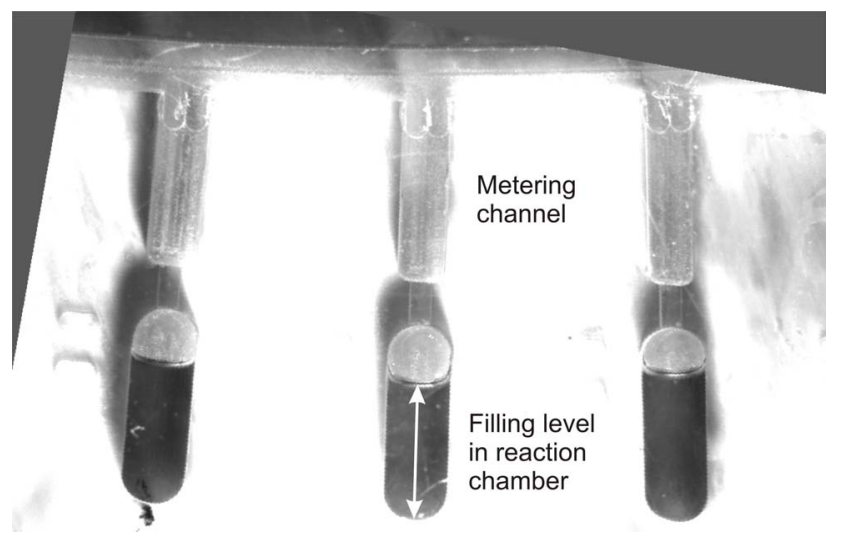

Figure 7: Camera image of filled reaction chambers. The filling levels are measured directly in the image, using the distance from the lowest point of the meniscus to the bottom of the reaction chamber.

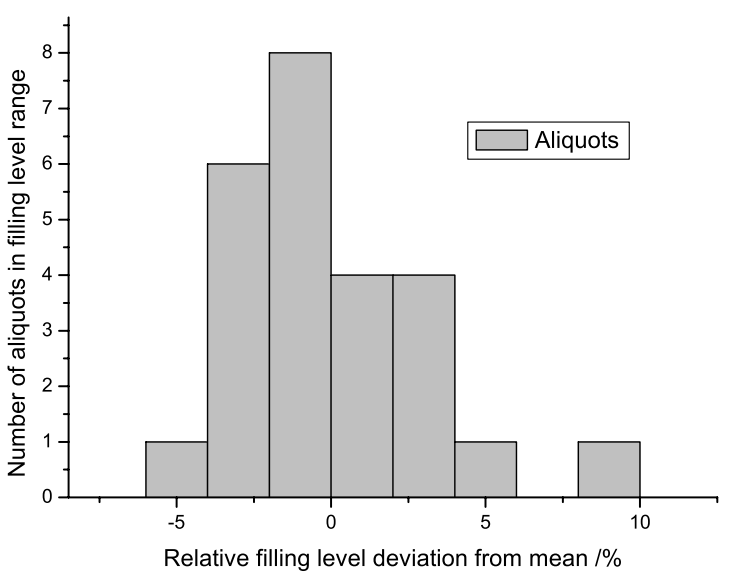

Figure 8: Histogram of the final filling levels in the reaction chambers. The relative difference from the mean filling level $(2.8 \mathrm{~mm})$ is shown.

\section{CONCLUSIONS}

We presented and experimentally validated a novel microvalve for centrifugal microfluidics which neither needs expensive local coatings nor micron-precise geometrical features. The valve enables an easy way to integrate valving features for assay automation in centrifugally driven lab-on-a-chip systems. The valve was utilized in a new aliquoting structure that allowed reliable aliquoting and metering.

\section{ACKNOWLEDGEMENTS}

We acknowledge financial support by the German Federal Ministry of Education and Research (project Zentrilab, link number V3BIO007) and the European Union (project MagRSA, contract No. 037957).

\section{REFERENCES}

[1] M. Madou, J. Zoval, G. Y. Jia, H. Kido, J. Kim, and N. Kim, "Lab on a CD," Annual Review of Biomedical Engineering, vol. 8, pp. 601-628, 2006.

[2] D. C. Duffy, H. L. Gillis, J. Lin, N. F. Sheppard, Jr., and G. J. Kellogg, "Microfabricated Centrifugal Microfluidic Systems: Characterization and Multiple Enzymatic Assays," Analytical Chemistry, vol. 71, no. 20, pp. 4669-4678, 1999.

[3] C. T. Schembri, T. L. Burd, A. R. Kopfsill, L. R. Shea, and B. Braynin, "Centrifugation and Capillarity Integrated Into A Multiple Analyte Whole-Blood Analyzer," Journal of Automatic Chemistry, vol. 17, no. 3, pp. 99-104, May1995.

[4] J. Ducrée, S. Haeberle, S. Lutz, S. Pausch, F. v. Stetten, and R. Zengerle, "The centrifugal microfluidic BioDisk platform," Journal of Micromechanics and Microengineering, vol. 17, no. 7, p. S103-S115, 2007.

[5] L. Chen, A. Manz, and P. J. R. Day, "Total nucleic acid analysis integrated on microfluidic devices," Lab on $A$ Chip, Aug.2007.

[6] N. Honda, U. Lindberg, P. Andersson, S. Hoffman, and H. Takei, "Simultaneous multiple immunoassays in a compact disc-shaped microfluidic device based on centrifugal force," Clinical Chemistry, vol. 51, no. 10, pp. 1955-1961, Oct.2005.

[7] Y. K. Cho, J. G. Lee, J. M. Park, B. S. Lee, Y. Lee, and C. Ko, "One-step pathogen specific DNA extraction from whole blood on a centrifugal microfluidic device," Lab on A Chip, vol. 7, no. 5, pp. 565-573, Feb.2007.

[8] D. H. Sharp, "An Overview of Rayleigh-Taylor Instability," Physica D, vol. 12, no. 1-3, pp. 3-18, 1984.

[9] M. S. Plesset and D.-Y. Hsieh, "General Analysis of the Stability of Superposed Fluids," Physics of Fluids, vol. 7, no. 8, pp. 1099-1108, 1964.

[10] J. Steigert, S. Haeberle, T. Brenner, C. Muller, C. P. Steinert, P. Koltay, N. Gottschlich, H. Reinecke, J. Ruhe, R. Zengerle, and J. Ducree, "Rapid prototyping of microfluidic chips in COC," Journal of Micromechanics and Microengineering, vol. 17, no. 2, pp. 333-341, Feb.2007. 